\title{
Virtually Transcribed and Restrained Robotic System
}

\author{
P. Choudesh Varma ${ }^{1}$, G. Venkateswarlu ${ }^{2}$, K.Uday $^{3}$ \\ ${ }^{1}$ Asst. Professor, CVR College of Engineering/EIE Department, Hyderabad, India \\ Email: choudesh82@gmail.com \\ ${ }^{2}$ Asst. Professor, CVR College of Engineering/EIE Department, Hyderabad, India \\ Email: venkigummadilli@gmail.com \\ ${ }^{3}$ Asst. Professor, CVR College of Engineering/EIE Department, Hyderabad, India \\ Email: kaparthiuday@gmail.com
}

\begin{abstract}
Robotic technologies are being used in various sectors for performing different tasks. In these technologies Pre defined actuator control programming techniques are used to perform desired tasks in the selected filed instead of human beings. But, Robotic control programming needs to be changed \& reconfigured to change the control action \& nature of working. So, there is no reliable technology to avoid reprogramming the robot. So, this paper presents development of artificial replicator to avoid reprogramming the robot for different situations, tasks such as bomb diffusion and sensitive surgery, taking readings from an active volcano or scientific analysis at radiation affected areas. In this proposed work, the wireless flexible gloves (emulator) are designed; which are integrated with flex, accelerometer, force, tilt, temperature sensors $\&$ also peltier modules and these sensors's output are connected to Arduino board \& calibrated into the real nature of signal using Arduino programming. This Arduino with WiFi shield communication is used to interact wirelessly with replicator-Robot. The desired control actions are recommended to robot by emulator moments through wearing gloves. The movements of robot hand wrist, fingers, forearm, head, elbow and shoulder can be controlled by advanced version Fx-5U PLC(Programmable Logic Controller).This PLC has the feature of industry internet protocol so emulator can be easily communicated with robot. Melsoft GX-Works-3 programming software is being to develop the algorithm for manipulation of emulator commands to actuator control signals. Here PLC executes actuator control Ladder programming with the speed of $40 \mathrm{~ns}$.
\end{abstract}

Index Terms: Robotics emulator, replicator, actuator, virtual, wireless glove.

\section{INTRODUCTION}

This robotics system is built both virtually and physically, which is controlled by human with replicate actions [1]. There is no direct human development. In this robotic system, parts of robot are similar to a human body(such as hands, fingers), which are controlled by natural movements of human body, and those movements are sensed through the glove worn by the human whose actions are to be replicated \& recorded for the further use. One human can control several hands by replicating these devices, which will be a breakthrough for many problems in the fields of Biomedical, Defense [2], Industry and manufacture of hand made goods. This proposal's first priority is to effectively create exact movements in the robot just as that of the person controlling it \& also other main features of this innovation, include feeling the object located miles away, perform human operations on the object, replicate human actions and repeat them when ever needed.

All the programming and effective controlling is done with the help of PLC (Programmable Logic Controllers) which is power saving, efficient and multifunctional. The special feature of the V-TARRS (Virtually -Transcribed and Restrained Robotic System) is that, it gives exact pressure and force, which are being applied through gloves at a particular location, these are read by replicator. For example cutting a vegetable, two different vegetables have two different structure and density, so V-TARRS Robot will be intelligently built [3] to apply appropriate pressures for appropriate tasks.

\section{System Design And Methodology}

V-TARRS basically have two different sections, one is transmission section \& another one is receiving section. Transmission section has wireless gloves with integration of sensors \& Wi-Fi shield. Receiving section has robot electro mechanical elements with PLC. V-TARRS can be divided into three major blocks,

A. Transcribed set up-replicator,

B. Programmed controller block (which includes PLC) and C. Emulator.

\section{A. Transcribed set up-replicator}

It is the robotic electro mechanical arrangement (shown in Figure1) which includes the physical replica of human hand and fingers with additional extensions which can extend robot's radius of reach, the robot's movements are made with the help of compressor pipes similar to the human nerve system in arms and with the help of the servo actuators mechanism. 2 Amps \& 5 Amps servo driver circuits are used to drive the shoulder movements of robot. On to the robotic hands, sensory elements are attached [4], which can detect the presence of the object, applied force \& it's temperature. Figure 2 represents the real-time developed model of robot-transcribed setup. The camera set up to this robot arrangement helps operator to properly see the surrounding of the robot. The data from these sensing elements \& actuators are connected to the PLC. This controller is communicated to the emulator through TCP (Transmission Control Protocol) and feature of TCP enables us to obtain the data between emulator \& replicator from anywhere around the globe. 


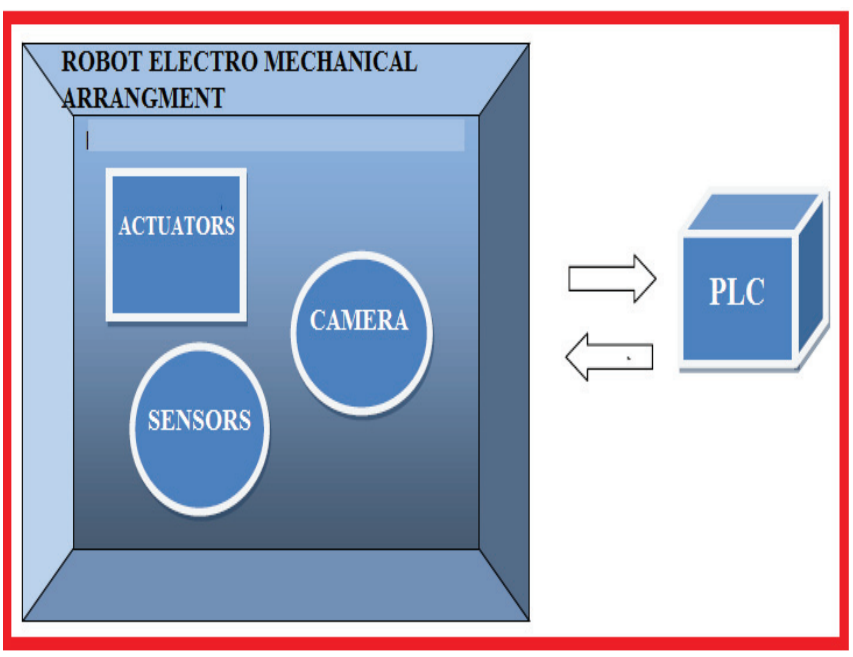

Figure 1. Elements of Transcribed setup

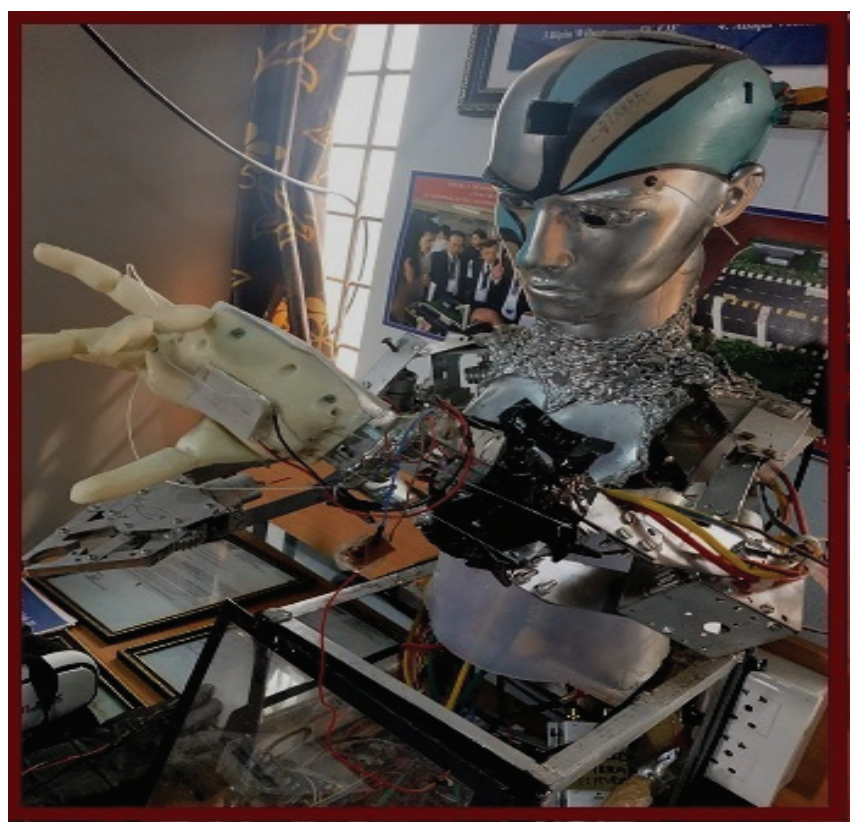

Figure 2. Developed model of Transcribed setup

\section{B. Programmed controller block}

This block is located at Transcribed set up. This block mainly performs the control operations on the actuators of replicator. PLC [5] ladder programming is used to write the programming for manipulation of emulator signal into control actions of robot. The Wi-Fi (transmitter + receiver) modules are located at the both ends, at the emulator side (to send the data to PLC and receive the transcribed set up's data), and one at the transcribed set up (for the robot to receive the data from the emulator and send data to emulator block).

\section{CASE 1: Medical surgery using V-TARRS technology}

i. In this case, if a person is needed to get operated immediately but the doctor is not available or he may be in the other location, then this technology comes into picture as shown in Figure3. ii. The doctor who is in other country or in any remote location he just sits in the emulator setup and he controls the robot present in the operation theatre.

The robot which presents their acts according to movements by the emulator (flexible gloves) and these robot movements can be observed by the doctor through a monitor.

iii. The PLC programming [6] is done in such a way that maximum accuracy is maintained and if there is any power cut during the process there are additional batteries placed which make the operation to continue.

\section{CASE 2: Bomb diffusion by armed forces:}

i. While diffusing a bomb [4] V-TARRS robot can be sent to the location and the robot can be operated by military person who is sitting in a distant location and the bomb can be diffused without putting himself into any risk through the emulator commands, which transmits the movements or actions of the operating person to the robot over internet protocol. This emulator is integrated with multiple sensors to detect the entire hand movements of the operator. This assures the life of those people $100 \%$.

ii. The robotic hands operate according to how the military personally operates and the whole operation can be viewed through a monitor as camera used in the V-TARRS.

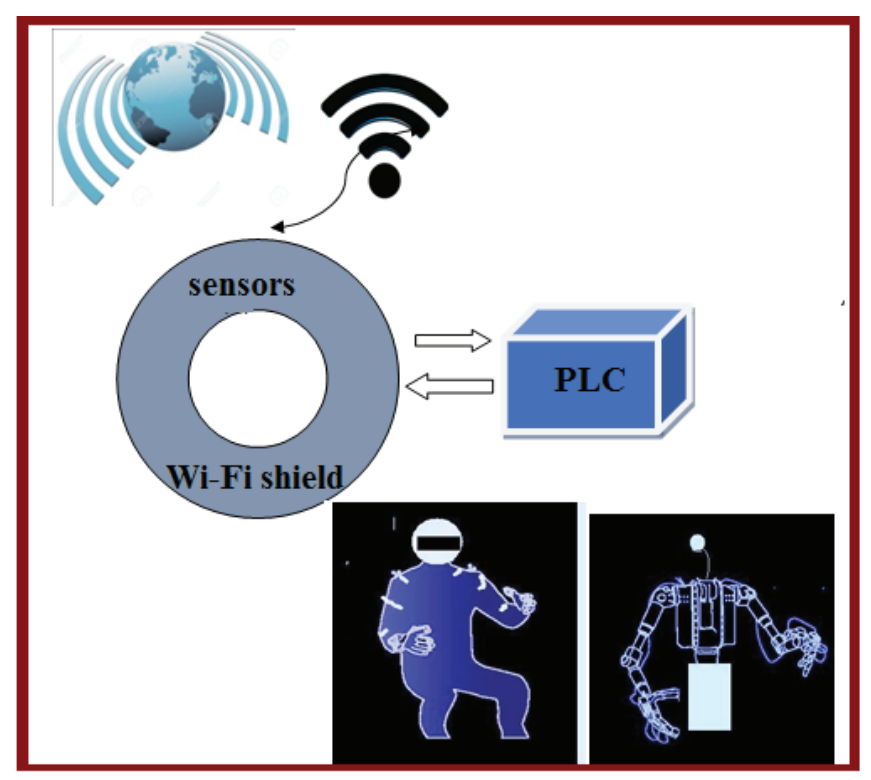

Figure 3. Graphical view of doctor operating patient using v-TARRS Technology

\section{Emulator block}

This is the area where human interaction is directly involved, human will wear gloves which will read the movements of fingers \& hand movements in all respective degrees of freedom as shown in Figure4, and this is possible by using the sensor elements like piezo resistive and force sensors. It also contains peltier module, which enables human feel the object which the robot (operator is controlling) is holding even from miles away. The HMI (Human Machine Interface) [7] enables us perform control operations on the robot while failure of glove sensors. 


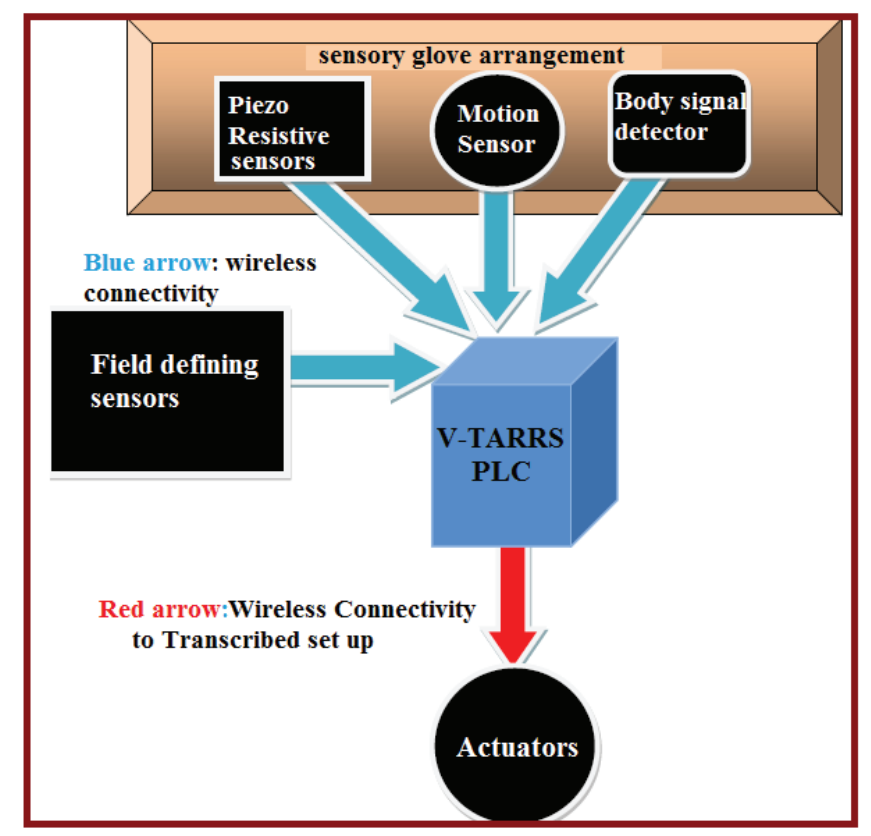

Figure 4. Elements of Emulator block.

\section{InNOVATION AND Theme Highlights}

\section{Problem statement}

- For bomb diffusing and sensitive surgery, human hand expertise is needed. Now, there is no reliable technology which can diffuse any kind of analog bombs.

- Although for the surgeries, robotic surgical technology is available, it requires a lot of pre operation calibration and settings are required, and in case of emergencies, wasting much time is unaffordable.

- Virtual robotic system is a design, which can be controlled both virtually and physically by humans, and replicate actions whenever needed and where ever want to apply, where humans cannot involve directly.

- Here the robotic system, parts of which are similar to human assets, is controlled by natural human arm movements.

- The movement is sensed through the glove worn by the human whose actions are to be replicated or recorded for the further use.

- This virtual robotic system can be interfaced with web technology application hence helping the person to perform operations quickly and effectively.

\section{Innovation}

- Here a new technology is being introduced which includes both human and robot technology.

- This is new because all the tasks cannot be performed by artificial intelligence itself, even if it is possible it may not be effective as human beings think instantaneously when compared to robot as robot intelligence is limited.

- So, by using this V-TARRS, the robot can be made to work according to human interference [4] from a remote location.

- By using wireless technology, precious lives of people can be saved, who daily involve in dangerous situations.

- If a person is of lack of time and he is immediately wanted to get operated but the doctor is in some distant place, then using this V-TARRS, the delay can be reduced.

\section{Theme highlights}

- V-TARRS could become an industrial revolution, where people cannot reach, V-TARRS can.

- Handloom industries which preserve national heritage can be uplifted.

- Industrial workers who have lost their limbs or legs but have required skill set might find employment again, they can work through robots they can walk through the simulation of V-TARRS.

- The filtering lung of V-TARRS purifies the air, foams placed in these lungs absorb harmful air components and this could prevent the industrial environment from getting polluted.

- V-TARRS will be helpful when the skill set of certain human is needed but where human cannot reach, V-TARRS can be sent as a substitute.

- V-TARRS, will surely make a huge impact once it's being used in Coal Mining, Handloom Industries, Semi-Automatic Power Plants, Chemical and Pharmaceutical Industries.

\section{HARDWARE \& SOFTWARE}

This section describes about major hardwares used like PLC, HMI, sensors, and Signal conditioning circuits. It gives detailed description of role of each \&specifications.

\section{Programmable Logic Controller:}

A PLC is a real-time system as outputs are changed according to inputs within bounded time otherwise unintended operation result occurs [10]. In this paper, the system sequence of operation is controlled by FX5U-32M PLC \& programmed by ladder diagram using MELSOFT GX works-3 software. Table.I represents the specifications of FX5U-32M PLC, Which have Consistency, Reliability, increased Productivity, Accuracy \& easy to modify wiring when control contents are changed.

\section{Features of GX WORKS3 MELSOFT}

$\begin{array}{ll}\checkmark & \text { Simple drag and drop } \\ \checkmark & \text { Flexible register programming } \\ \checkmark & \text { Easy visualization and confirm changes in program } \\ \checkmark & \text { Motion control CPU hardware can be simulated } \\ \checkmark & \text { Easy maintenance } \\ \checkmark & \text { Reduction in downtime and high productivity. } \\ \checkmark & \text { Easy error rectification and monitoring. }\end{array}$


TABLE I.

SPECIFICATIONS OF FX5U 32M-PLC

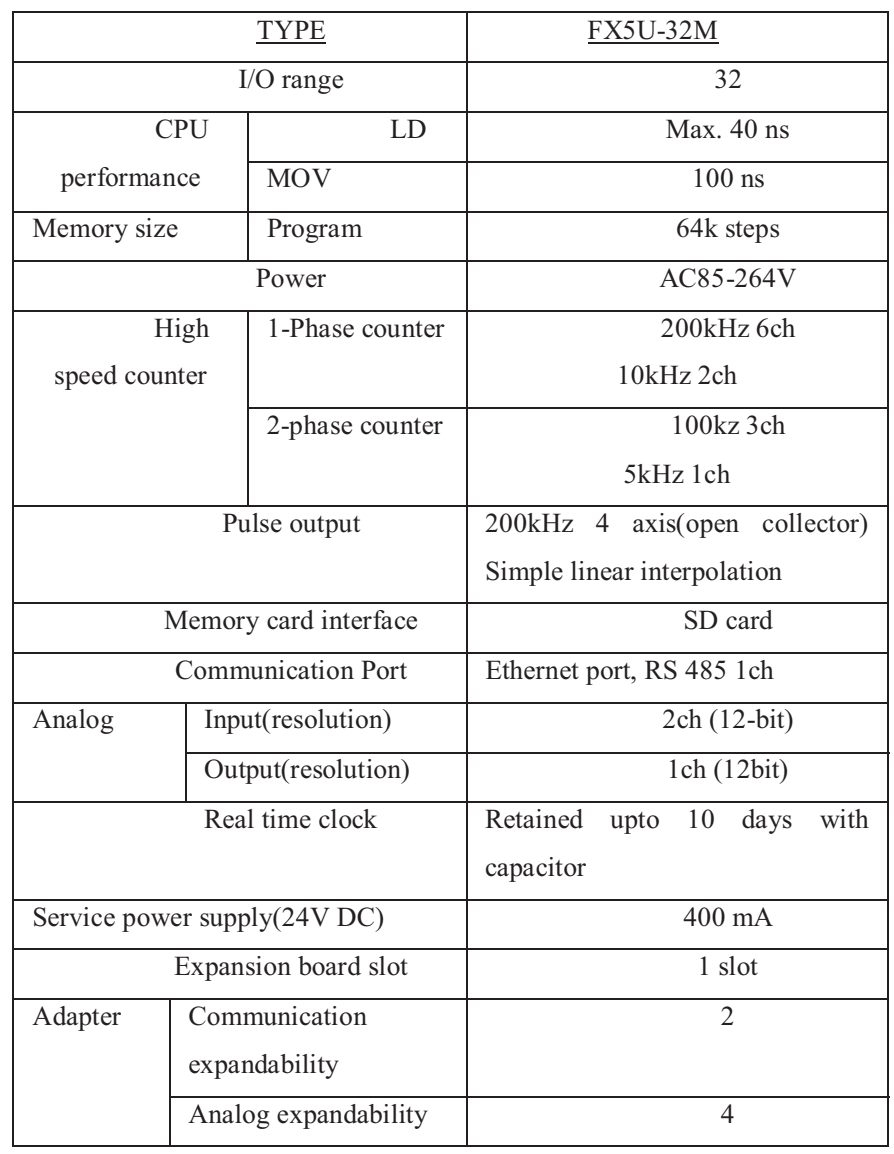

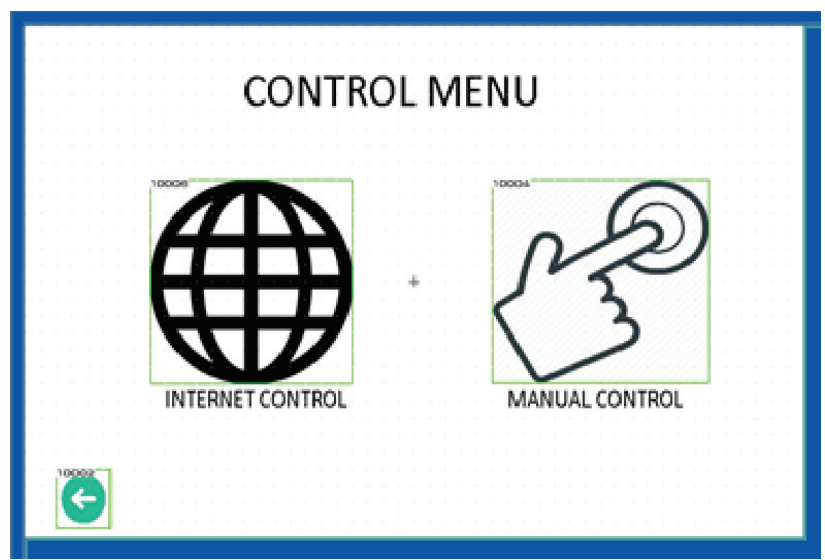

Figure 6. Operation that can be performed using HMI

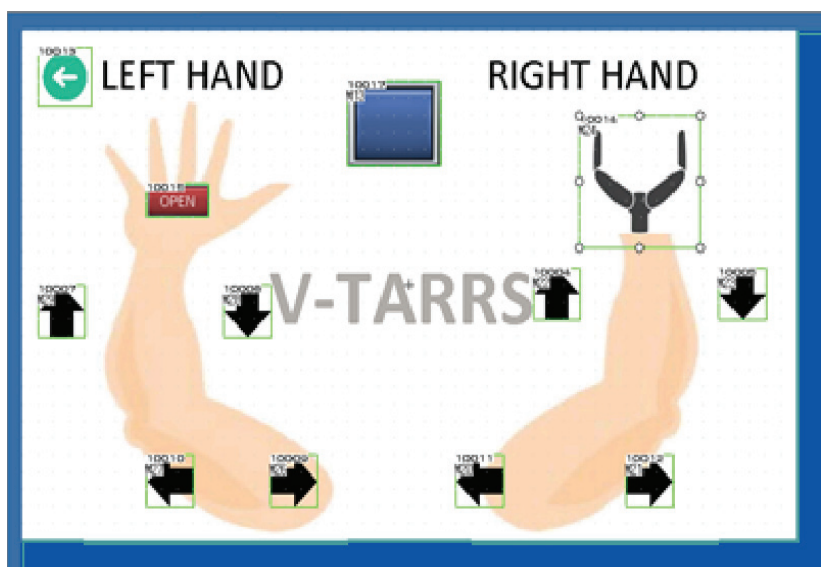

Figure 7. Manual Control in various directions

In this system, operators are also allowed to control the robot actions through Graphical Operating Terminal (GOT) while predefined actions are required. Developed graphical screens are shown from Figure 5 to Figure 7. Some limited actions only can be done by the operator through graphical operating terminal or Human Machine Interface (HMI). GT Designer 3 software is used for the development of HMI screens [8].

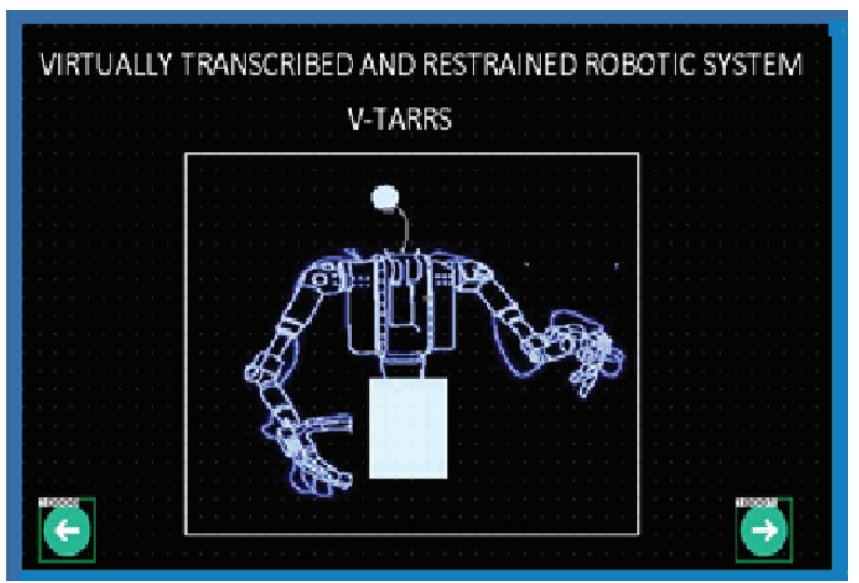

Alternative approach to analog to digital converter

As FX-5U PLC is used for calibration \& control applications, it has only two analog inputs. But in this proposed work, many sensors are being used whose output is of analog in nature. In order to make PLC [9] understand this analog signal, Voltage Controlled Oscillator (VCO) is used to produce digital output i.e. converting analog signal into frequency signal and are making PLC to read those frequency signals using SPD (Special Programming Device) High speed function.

Here, JMK wireless camera module is being used (shown in Figure8) for monitoring and one more camera are being used is webcam for video conference. This wireless camera is adjustable for 50 to 100 meters' communication range. It is mainly suitable for small size mobile robots. Image can be transmitted wireless acquired by the robot to the PC and do the image processing as well as robot actuator control. It can work in wireless and wired mode. Wireless camera is powered by $9 \mathrm{~V}$ battery or $9 \mathrm{~V}$ DC adapter. Receiver can be powered by $9 \mathrm{~V} / 12 \mathrm{~V}$ DC adapter.

Figure 5. Basic display of HMI screen 


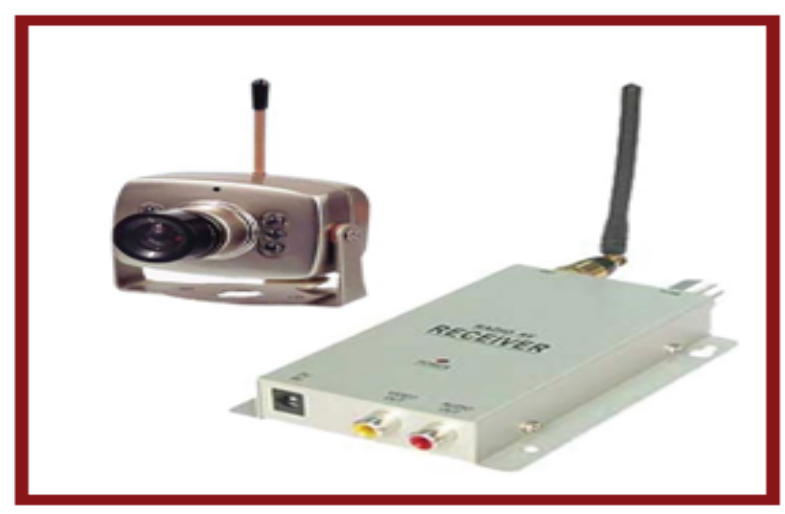

Figure 8. Wireless Camera and Receiver module

Specifications of force sensors

1. Size: $1 / 2^{\prime \prime}(12.5 \mathrm{~mm})$ diameter active area by $0.02 "$ thick.

2. Resistance range: Infinite/open circuit (no pressure), $100 \mathrm{~K} \Omega$ (light pressure) to $200 \Omega$ (max. pressure).

3. Force range: 0 to $20 \mathrm{lb}$. (0 to 100 Newtons) applied evenly over the $0.125 \mathrm{sq}$ in surface area.

4. Power supply: Uses less than $1 \mathrm{~mA}$ of current (depends on any pullup/down resistors used and supply voltage).

Flex sensors

Flex sensors also called as bend sensors, which measure the amount of deflection caused by bending of human fingers, wrist moments through gloves. There are various ways of sensing deflection, from strain-gauges to hall-effect sensors. Signal Conditioning Circuit (SCC) is required to convert the change of the resistance into voltage [10] with respect to the change of movements of the wearied gloves.

The impedance buffer in the Basic flex sensor circuit, (SCC-Figure 9) is a single sided operational amplifier, which is used with these sensors because the low bias current of the op amp reduces error due to source impedance of the flex sensor as voltage divider. A potentiometer can be added to the circuit to adjust the sensitivity range.

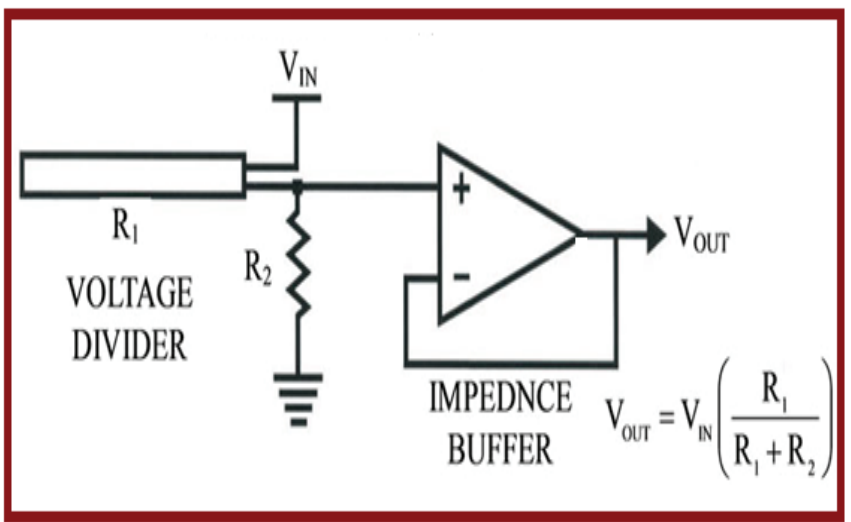

Figure 9. Basic Flex sensor signal conditioning circuit

\author{
Mechanical Specifications: \\ -Life Cycle: $>1$ million \\ -Height: $0.43 \mathrm{~mm}\left(0.017^{\prime \prime}\right)$ \\ -Temperature Range: $-35^{\circ} \mathrm{C}$ to $+80^{\circ} \mathrm{C}$. \\ Electrical Specifications:
}

-Flat Resistance: 25K Ohms

-Resistance Tolerance: $\pm 30 \%$

-Bend Resistance Range: 45K to $125 \mathrm{~K}$ Ohms (depending on bend radius)

-Power Rating: 0.50 Watts continuous. 1 Watt Peak.

\section{Arduino with $\mathrm{Wi}$-Fi shield}

In this proposed work, Arduino Uno is being used to calibrate the Flex, tilt, force \& proximity sensors, which are integrated with gloves to detect the various movements of hand as shown in Fig.10.Each glove is integrated with six flexible sensors, one tilt sensor, five force sensors \& one proximity sensor.

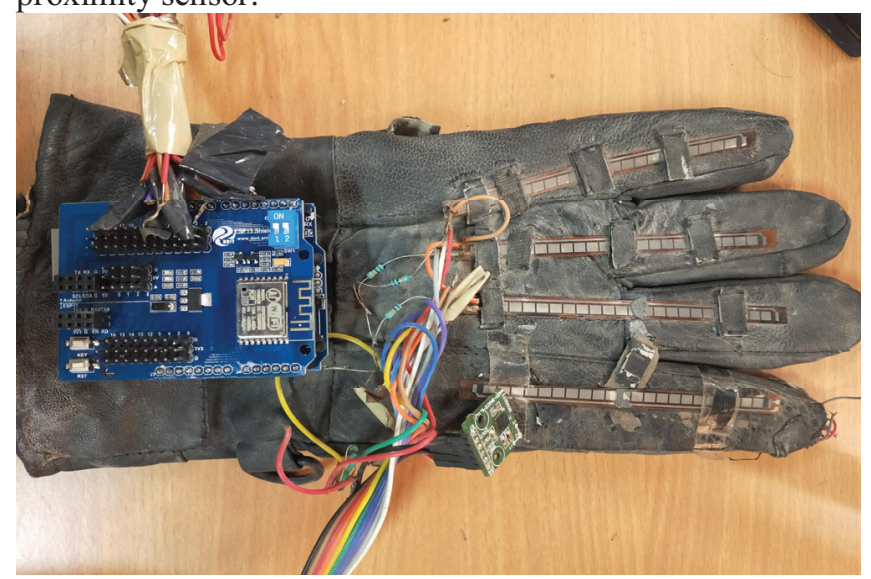

Figure 10. Emulator with Arduino \& integrated sensors

All these sensors output is calibrated into the real nature of the signal by using special programming software Arduino IDE. The calibrated data is to be transmitted to the robot or Transcribed block wirelessly. So in this work, low-cost WiFi shield-WizFi210 is connected with Arduino uno board through the serial communication protocol. It operates with standard $802.11 \mathrm{~b} / \mathrm{g} / \mathrm{n}$ access points at speed up to $11 \mathrm{Mbps}$. Where WizFi210 shield has been set to act as a TCP/IP server to communicate robot $\&$ address of server is maintained as 192.168.43.150.

\section{IoT APPLICATION}

Here, UBIDOTS cloud connectivity is being used for wireless and IoT (Internet of Things) application. With the help of this cloud, data can be sent and received wirelessly. It is an open source IoT platform. It also stores the previous actions \& manipulations done by the operator [11]. In this UBIDOTS, variable actions are created and assigned these actions to the actuators as shown in Figure 11. Sensor readings also can be monitored through these variables. A code is written in C Language for sending and retrieving the data from the variables. 

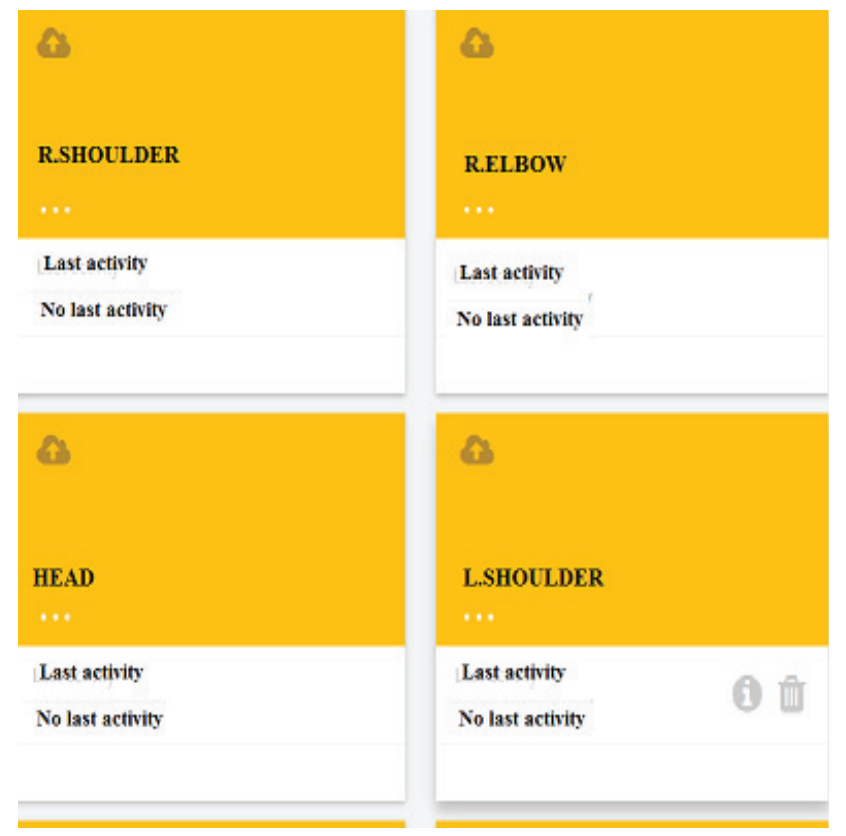

Figure 11. Variables created in UBIDOTS

\section{Results}

Emulator has been designed in the desired configurations \& established communication successfully between emulator \& transcribed block by TCP protocol. Different movements \& commands were given through emulator by wearing gloves, where robot replicated all the movements given by emulator as shown in figure 12 \& 13. This proposed work has been implemented experimentally in the automation laboratory. In this work, various parameters were recorded like force applied by emulator \& replicator on the object, linearity of flex sensors with respect to replicator movements. The comparison of force generated on the object by fingers of emulator \& replicator produces $\pm 1.3 \%$ to $\pm 1.7 \%$ range of the error $(98.3 \%$ to $98.7 \%$ range of accuracy). These errors are mainly caused by Hysteresis losses generated by replicator servo mechanisms. Table. II \& Table.III represent the numerical comparison of forces generated on object by various fingers of emulator \& replicator and it produces $\pm 1.4 \%$ of linearity.

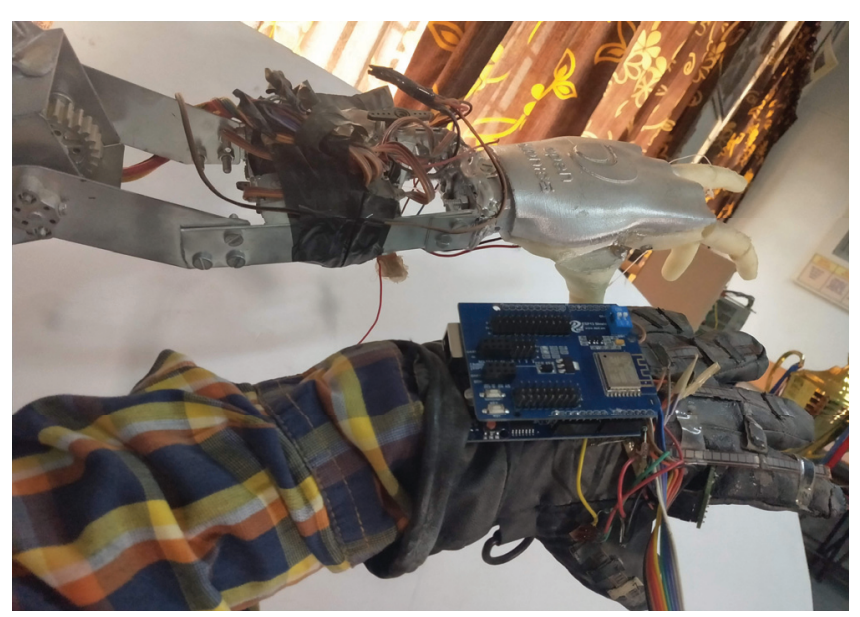

Figure 12. Right hand movement of emulator \& replicator

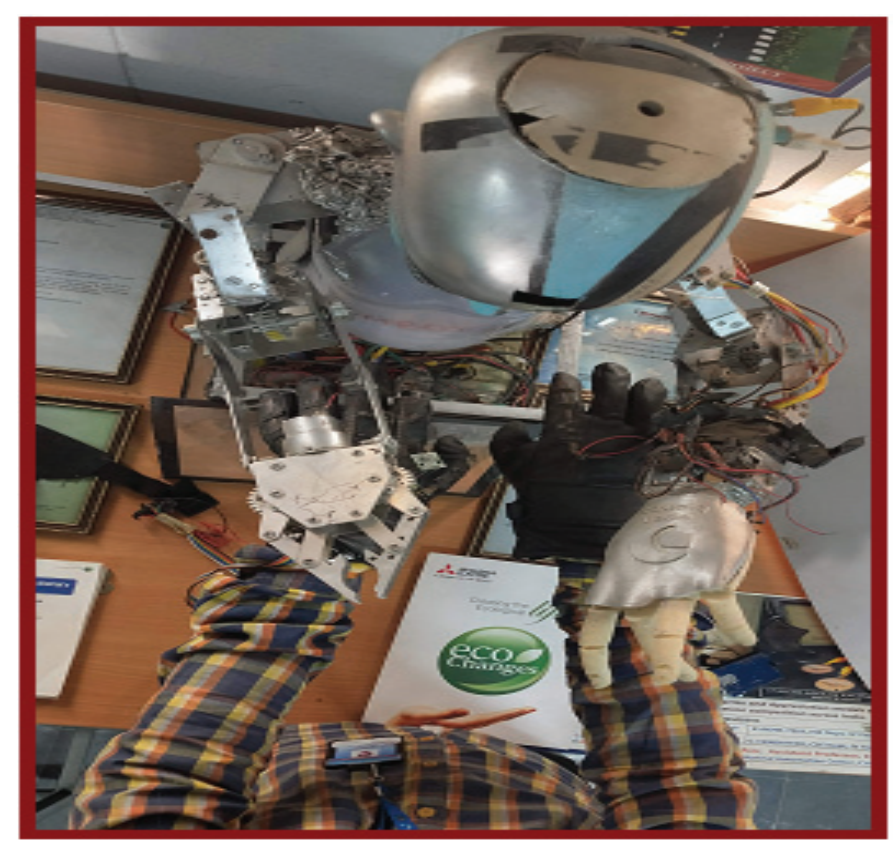

Figure 13. Two hands movement of emulator \& replicator

TABLE III.

FORCE COMPARISON OF EMULATOR \& REPLICATOR-LEFT HAND

\begin{tabular}{|c|c|c|c|c|c|}
\hline \multicolumn{7}{|c|}{ Force (Newton) measurement, left hand-Emulator } \\
\hline & $\begin{array}{c}\text { Little } \\
\text { finger }\end{array}$ & $\begin{array}{c}\text { Ring } \\
\text { finger }\end{array}$ & $\begin{array}{c}\text { Middle } \\
\text { finger }\end{array}$ & $\begin{array}{c}\text { Index } \\
\text { finger }\end{array}$ & Thumb \\
\hline Average & 38.2 & 45.4 & 63.6 & 64.0 & 122.3 \\
\hline \multicolumn{7}{|c|}{ Force measurement, left hand (N)-Replicator } \\
\hline & Little & Ring & Middle & Index & Thumb \\
& finger & finger & finger & finger & \\
\hline Average & 38.73 & 46.4 & 62.77 & 63.13 & 120.3 \\
\hline Error & $+1.40 \%$ & $+1.40 \%$ & $-1.30 \%$ & $-1.35 \%$ & $-1.62 \%$ \\
\hline
\end{tabular}

TABLE III.

FORCE COMPARISON OF EMULATOR \& REPLICATOR-RIGHT HAND GRIPPER

\begin{tabular}{|c|c|c|}
\hline \multicolumn{3}{|c|}{ Force (Newton) measurement, Right hand gripper-Emulator } \\
\hline & finger-1 & finger-1 \\
\hline Average & 110.2 & 109.2 \\
\hline \multicolumn{2}{|c|}{ Force measurement, right hand gripper (N)-Replicator } \\
\hline & Gripper finger-1 & Gripper finger-2 \\
\hline Average & 108.7 & 107.7 \\
\hline Error & $-1.30 \%$ & $1.37 \%$ \\
\hline
\end{tabular}

\section{Future Scope \& APplications}

This proposed work has wide range of applications, it can be used in military for bomb diffusing, risk firing in the space missions, surgeries, handmade good production, and it can be applied in industries for smart production where the human actions have to be repeated especially in the areas where automation is not available. In the future by using 
these kind of devices like V-TARRS, physically challenged people can be helped to overcome daily challenges by making V-TARRS controlled through voice commands. Physically challenged people can simply move the artificial limbs just by speaking to the V-TARRS, or by connecting it to nervous system. BIO METRIC protection can be implemented and V-TARRS can be secured and limited to only one person.

\section{CONCLUSIONS}

Interfacing of flex, accelerometer and other sensors to Arduino \& PLC along with signal conditioning circuits was successfully accomplished and acquired data from field devices has been calibrated into physical movements using SPD (Special Programming Device), PWM (Pulse Width Modulation) and basic ladder functions in PLC. The prototype servo motors for driving robotic arm elbow, wrist \& finger movements were successfully driven by PLC output module with the help of PWM programming functions. HMI screen layouts have been developed and the wireless network communication has been successfully established between emulator \& replicator. Enough efforts were carried out to establish Internet of Things using Arduino with Wi-Fi shield.

\section{REFERENCES}

[1] H.Oyama and F.Wako "Evaluation of a virtual reality system for medicine" IEEE International conference on virtual systems \& Multimedia, Geneva, Switzerland, 06 August 2002.

[2] Abdul Kadir Bin Motaleb, Mohammad Busayeed Hoque, and Md. Ahsanul Hoque "Bomb disposal robot". International Conference on Innovations in Science, Engineering and Technology (ICISET), IEEE publisher, Dhaka, Bangladesh, 16 February 2017.

[3] I. H. Lin, C. Y. Liu, and L. C. Chen, "Evaluation of HumanRobot Arm Movement Imitation", Proceedings of 8th Asian Control Conference (ASCC), PP. 287-292, 2011.

[4] Takumi Kawasetsu, Takato Horii, Hisashi Ishihara, and Minoru Asada "Flexible Tri-Axis Tactile Sensor Using Spiral Inductor and Magneto rheological Elastomeric" IEEE Sensors Journals, volume18, PP: 5834 - 5841 July 15, 2018.

[5] Bolton, W, "Programmable logic controllers", Access Online via Elsevier, 2009.

[6] Skulavik Tomas, Kopcek Michal, and Kopeekova Alena "Fuzzy control of robotic arm implemented in PLC", 9th International Conference on Computational Cybernetics (ICCC),IEEE,Publisher, Tihany, Hungary, 03 October 2013.

[7] AmarBanerji, M. Tabassum, D. Venkatesh, and Manjit Singh."An interactive GUI for dual-robot command console" Annual IEEE India conference, volume 1, 2008.

[8] Lai Wei and Huosheng Hu" EMG and visual based HMI for hands-free control of an intelligent wheelchair", World Congress on Intelligent Control and Automation, IEEE Publisher Jinan, China, 7-9 July 2010.

[9] Chung, C. A, 'A cost-effective approach for the development of an integrated pc-plc-robot system for industrial engineering education', IEEE Transactions on Education, Volume: 41, Issue: 4 ,PP- 306-310, Nov 1998 .
[10] D.Roy Choudhury and Shail B Jain, "Linear Integrated Circuits", 2nd Edition, New Age International (P) Limited, PP-56-58.

[11] Federico Montore, Luca Bedogu, and Luciano Bononi”A collaborative Internet of things Architecture for smart cities and Environmental monitoring",.IEEE Internet of things Journal, volume: 5,issue 2,PP- 592 - 605 ,April 2018. 